\title{
The Effect of Educational Intervention on Improving Health Literacy Skills Among Dormitory Students of Shahid Beheshti University of Medical Sciences, Tehran, Iran
}

\author{
Rahman Panahi ${ }^{1}$, Fereshteh Osmani ${ }^{2}$, Malihe Pishvaei ${ }^{3}$ and Shamsaddin Niknami ${ }^{1, *}$ \\ ${ }^{1}$ Department of Health Education \& Health Promotion, Faculty of Medical Sciences, Tarbiat Modares University, Tehran, Iran \\ ${ }^{2}$ Department of Biostatistics, Faculty of Medical Sciences, Tarbiat Modares University, Tehran, Iran \\ ${ }^{3}$ Department of Family Social Health, Social Development and Health Promotion Research Center, Gonabad University of Medical Sciences, Gonabad, Iran \\ "Corresponding author: Department of Health Education \& Health Promotion, Faculty of Medical Sciences, Tarbiat Modares University, Tehran, Iran. Tel: +98-2182883869, \\ Email: niknamis@modares.ac.ir
}

Received 2018 June 09; Accepted 2019 April 08.

Keywords: Students, Health Literacy, Skills, Intervention

\section{Dear Editor,}

Health literacy (HL) is related to literacy and consist of the knowledge, motivation, and the capacity of individuals to access, understanding, evaluating and the use of health information in order to judge and make routine decisions about health care, disease prevention, and health promotion to maintain or enhance the quality of life (1). Because there was no clear evidence whether the HL of students of medical sciences is at a desirable level, several studies were carried out among the students of Shahid Beheshti University of Medical Sciences in Tehran, which indicated that there was a wide range of inadequate and marginal $\mathrm{HL}$ (68.8\%) and inadequate and problematic HL (37.2\%) among them $(2,3)$.

In recent years, the student population of Iran has grown significantly. Therefore, it is important to recognize the effective factors in adapting the students to more healthy behaviors and reducing risk behaviors (2). The students were selected based on two criteria: first, their age and their training, and second, the pattern of a healthy lifestyle in the society, especially students of medical sciences who can effectively promote the public health (2). Given the wide range of the prevalence of inadequate, problematic, and marginal HL among medical students $(2,3)$, and the effect of HL on increasing health behaviors and improving access to health care (4); the purpose of the present study was to determine the effect of educational intervention on improving HL skills among dormitory students of Shahid Beheshti University of Medical Sciences, Tehran, Iran between 2016 and 2017.

The present study was a quasi-experimental interven- tional study. The statistical population in this study was dormitory students of Shahid Beheshti University of Medical Sciences, Tehran, that 130 subjects (65 in each intervention and control group) were selected among them by multi-stage randomization. The inclusion criteria were the willingness of people to enter the study, having Iranian citizenship, students who were undergraduate (2nd or 3rd year of study), and lived in the dormitories affiliated to Shahid Beheshti University of Medical Sciences, Tehran. Also, incompletely filling the questionnaire was considered an exclusion criterion.

The data collection tool was a HELIA standard questionnaire for measuring HL that this questionnaire contains 33 questions and consists of 5 main dimensions, including reading, access, understanding, appraising, and decision making and application of health information and in the three stages of before, immediately, and three months after the intervention, which were completed by the students in both groups. In the present study, an educational intervention was performed using Telegram application and educational messages were sent to the students of the intervention group in 6 sessions. The collected data were analyzed using SPSS software version 16 . The normal distribution of the data was determined using the KolmogorovSmirnov test. For this reason, the groups were evaluated for changing the dependent variable by analyzing the variance of the repeated measure analysis, and the intervention group was compared with the control group at each stage of the evaluation; for quantitative variables the independent $t$-test and for evaluating the qualitative variables (demographic and background variables) the chi-square 
test were used. In this study, the significance level was considered 0.05 .

The results showed that there was no significant difference between the demographic and background variables and the mean scores of HL skills and total HL in the two groups before the intervention $(\mathrm{P}>0.05)$, but after the intervention, the comparison of the two groups showed that the mean scores of HL skills and total HL skills in the intervention group were significantly different in comparison to the control group $(\mathrm{P}<0.05)$. Also, the results indicated that in the intervention group during the study period, changes in the scores of HL skills and HL total were significant and parallel to the effect of training, while in the control group, the scores of these variables showed no significant change.

In general, the results of this study showed that educational intervention using Telegram can promote HL skills in the students. It seems that social networks, including
Telegram, can be effective in engaging students in improving their HL skills owing to their popularity among them.

\section{References}

1. Vozikis A, Drivas K, Milioris K. Health literacy among university students in Greece: Determinants and association with self-perceived health, health behaviours and health risks. Arch Public Health. 2014;72(1):15. doi: 10.1186/2049-3258-72-15. [PubMed: 24987522]. [PubMed Central: PMC4066308].

2. Ramezankhani A, Ghafari M, Rakhshani F, Ghanbari S, Azimi S. [Comparison of health literacy between medical and non-medical students in Shahid Beheshti Universities in the academic year 92-93]. Pajoohande. 2015;20(2):78-85. Persian.

3. Panahi R, Ramezankhani A, Tavousi M, Osmani F, Ghazanfari E, Niknami S. Evaluation of health literacy and its influencing factors on dormitory students of Shahid Beheshti University of Medical Sciences in Tehran. J Educ Commun Health. 2017;3(3):30-6. doi: 10.21859/jech03035.

4. Manganello JA. Health literacy and adolescents: A framework and agenda for future research. Health Educ Res. 2008;23(5):840-7. doi: 10.1093/her/cym069. [PubMed: 18024979]. 